\title{
REVISED Road traffic injuries in Nepal during COVID-19
}

\section{lockdown [version 3; peer review: 2 approved]}

\author{
Bhagabati Sedain (iD), Puspa Raj Pant (iD)2,3
}

${ }^{1}$ Department of Population Studies, Padma Kanya Multiple Campus, Tribhuvan University, Kathmandu, Bagmati, 44600, Nepal

${ }^{2}$ Centre for Academic Child Health, University of the West of England, Bristol, UK

${ }^{3}$ Nepal Injury Research Centre, Bhaktapur, Nepal

V3 First published: 08 Oct 2020, 9:1209

https://doi.org/10.12688/f1000research.26281.1

Second version: 14 Dec 2020, 9:1209

https://doi.org/10.12688/f1000research.26281.2

Latest published: 01 Feb 2021, 9:1209

https://doi.org/10.12688/f1000research.26281.3

\section{Abstract}

Background: As the world is busy addressing COVID-19, road traffic injuries, another major cause of death is continuously killing people on the roads. In Nepal, there were frequent media reports of occurrences of road crashes, injuries, and deaths despite nationwide lockdown. This paper aims to describe the situation of road traffic crashes and casualties during the period of complete lockdown.

Methods: This study used secondary data from two sources: Nepal Police and media reports between 24 March and 14 June 2020 (because the government lifted the nationwide lockdown from 15 June 2020). Available details of crashes, deaths, and injuries for this period were extracted from media reports and the summary data that was obtained from the Police. We have included data from both sources in the results.

Results: Nepal Police recorded 1,801 incidents of road crashes during the 82 days of the COVID-19 lockdown with 256 deaths (on average 3.1 deaths daily) and 1,824 injuries (on average 22.2 injuries daily). Motorcycles comprised over $21 \%$ of all vehicles involved in crashes. Ambulances and other vehicles for essential services were also found to be involved in crashes. Speeding itself was the cause for almost a quarter of the incidents during the lockdown.

Conclusions: Although a reduction in the number of road crashes and related injuries and fatalities was observed, this reduction was not as substantial as anticipated during the heavy restrictions on vehicular movement imposed during the lockdown. Media reports were mainly found to be reporting the crashes where deaths occurred, but police records also included nonfatal injuries. The incidence of crashes in this period shows that it is important to work for road safety to save lives from road traffic crashes in Nepal.

Keywords

COVID-19, Lockdown, Road Traffic Crashes, Injuries, Deaths

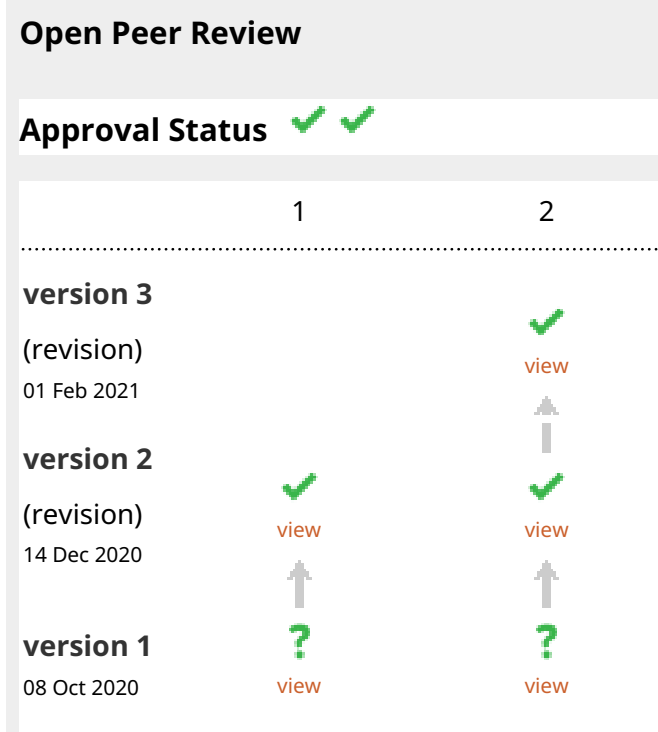

1. Kulanthayan KC Mani, University Putra Malaysia (UPM), Serdang, Malaysia

2. Felix Wilhelm Siebert ID, Friedrich Schiller University Jena, Jena, Germany Any reports and responses or comments on the article can be found at the end of the article. 


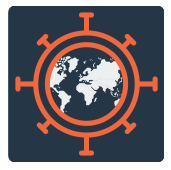

This article is included in the Emerging Diseases

and Outbreaks gateway.

collection.

Corresponding author: Bhagabati Sedain (bssedhai@gmail.com)

Author roles: Sedain B: Conceptualization, Data Curation, Formal Analysis, Methodology, Resources, Software, Visualization, Writing Original Draft Preparation; Pant PR: Conceptualization, Supervision, Writing - Review \& Editing

Competing interests: No competing interests were disclosed.

Grant information: The author(s) declared that no grants were involved in supporting this work.

Copyright: (c) 2021 Sedain B and Pant PR. This is an open access article distributed under the terms of the Creative Commons Attribution License, which permits unrestricted use, distribution, and reproduction in any medium, provided the original work is properly cited.

How to cite this article: Sedain B and Pant PR. Road traffic injuries in Nepal during COVID-19 lockdown [version 3; peer review: 2 approved] F1000Research 2021, 9:1209 https://doi.org/10.12688/f1000research.26281.3

First published: 08 Oct 2020, 9:1209 https://doi.org/10.12688/f1000research.26281.1 


\section{REVISED Amendments from Version 2}

There are no such major changes in this new version. Only the first sentence of the conclusion section of the abstract was reworded. Similarly, in the result section of the main text in Figure 3, a y-axis level was added and the figure description was revised.

Any further responses from the reviewers can be found at the end of the article

\section{Background}

The world experienced a series of unprecedented events since December 2019 after the detection of the Novel Coronavirus disease (COVID-19) (Asian Development Bank, 2020). The World Health Organisation (WHO) declared it a worldwide pandemic on 11 March 2020 (World Health Organization, 2020). During this period, social-distancing and lockdowns were implemented throughout the world. As of 14 June 2020, the spread of COVID-19 has reached all countries and territories around the globe with 282,733 deaths (Worldometer, 2020)

The concept of restrained movement and physical distancing is believed to support the breaking of the chain of infection (World Health Organization, 2014) and slowing the spread of the virus by limiting contact with infected people and contaminated surfaces. In many countries, everyone but essential workers have been instructed to stay at home and work from home. Consequently, transportation through all means has reduced in a never-before-seen manner. There are also reports of improvement in air quality (Wang et al., 2020) and reduced bed occupancy for road crash trauma in emergency departments (Zhu et al., 2020), which might have enabled health service systems to prepare and cope with a sudden rise in the number of COVID-19 hospitalisation. However, keeping people at home was not an easy job; governments had to impose notices with strict provisions - including fines and potential imprison if their decisions were violated.

Nepal also joined the global practice for the prevention of the spread of COVID-19 by declaring a ban on long-distance public travels from 22 March 2020 through the Prime Minister's statement to the nation. "All international flights coming to Nepal have been suspended effective from March 22 until 31. Effective from March 22, long-distance passenger vehicles will be suspended throughout Nepal for some time. Crowded places like cinema halls have been shut down for the time being." Prime Minister KP Sharma Oli, 20 March 2020 (Embassy of Nepal, 2020).

Within the window of the partial lockdown (21 March to $23 \mathrm{March}$ ), an estimated 1.5 million residents left the capital Kathmandu for different parts of the country. Similarly, about half a million migrant workers from India also returned to their homes in the wake of the government's decision to lock down the country (Pokhrel \& Awale, 2020). This resulted in a sudden rise in the use of motorised vehicles during the 21st, 22nd, and 23rd of March. Meanwhile, the second case of COVID-19 was detected on 23 March (Figure 1). Only then did the Government decided to impose a countrywide complete lockdown, from 24 March 2020 (Figure 1) (Budhathoki, 2020). Hence, the country's efforts and resources converged towards the prevention of coronavirus transmission.

However, the government authorised a special pass-permit to use private vehicles and motorcycles in case of an emergency. Only vehicles required for essential services, i.e. ambulances, police, fire service, milk-tankers, water-tankers, and food deliveries, were allowed on the road without the pass. Due to these activities, a sudden decline in vehicular movement was observed in Nepal. Subsequently, a large reduction in the number of crashes and casualty was expected during this lockdown. Unfortunately, there were frequent media reports of road crashes, injuries, and deaths despite the nationwide lockdown.

This paper aims to describe the situation of road traffic crashes and the subsequent casualties during the period of COVID-19 lockdown using secondary sources of data. In the study, we have presented the results from the two data sources to provide maximum possible details complemented by one-another.

\section{Methods}

This study utilised two secondary data sources, i.e. media reports, and published or unpublished police records. Data collection was done in two ways: a daily online search of media reports for vehicle crash incidents on Google, which was done using search terms in the Nepali language in order to capture most of the reports across the country. The search terms were "deaths or injuries", "road crash", "car crash", "motorcycle crash", "vehicle crash", "pedestrian hit by", "bicyclist hit by", "ambulance crash", "tractor crash", "truck crashes", or "crashes or collisions occurred during lockdown;". Similarly, data was also collected by contacting the police to obtain road crash records. From both these data sources, only limited number of

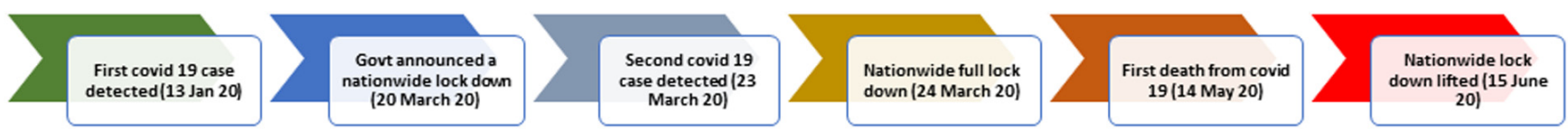

Figure 1. Timeline of COVID-19 related event in Nepal. Sources: Box 1 First covid case: Bastola et al., 2020. Box 2 lockdown announcement: Embassy of Nepal, 2020. Box 3 Second covid 19: Dhakal \& Karki, 2020. Box 4 Full Lock down: Mahato et al., 2020. Box 5 first covid 19 death: Poudel, 2020. Box 6 Lockdown lifted: Kharel, 2020. 
variables could be extracted. The location of crash, the vehicles, animals, people, or objects involved, the resultant number of deaths \& injuries, and the age and gender of victims were extracted from media reports into an Excel spreadsheet. The total number of crashes, deaths and injuries occurring in districts and provinces were taken from police records. This paper includes the road crash information for 82 days of the lockdown (24 March to 14 June 2020) from media reports and police records. The exact location and types of vehicles involved in fatal crashes were not available from the Police data, therefore the exact details of the vehicles and the location of crashes were extracted from the media reporting. With the available information on fatal crashes for Palika level (local government unit), the cases were nationally mapped for these units. In this study, we have presented the results from the two data sources to provide maximum possible details complemented by one another.

\section{Results}

Altogether, there were 1,801 incidents of road crashes recorded by the traffic police in 82 days (24 March to 14 June 2020) of lockdown from all seven provinces of Nepal, which included 2,602 vehicles (96\% motorized) that claimed 256 lives and led to a further 1,824 injuries (among which $32 \%$ were severely injured). However, the media mostly reported fatal crashes, as 200 deaths and 322 injuries were extracted through media reports for the same period. The number of deaths and injuries reported by local media and taken from police records are given in the Underlying data (Sedain \& Pant, 2020).

In this lockdown, no vehicles were allowed to operate without a government-issued pass for essential services. Police records show that in 82 days of full lockdown, an average of 3.1 people died and 22.2 people were injured daily as a result of road crashes. The media reporting of fatal road crashes was $21.8 \%$ less than the police record, and very few injuries and vehicle crashes were reported (Table 1).

Kathmandu Valley comprises of three districts, namely Kathmandu, Bhaktapur, and Lalitpur. Nepal Police has not recorded the road traffic deaths separately for these three districts and the records of the crashes were presented for Kathmandu Valley as a whole. Therefore, by including the three districts of Kathmandu Valley, Table 2 displays road traffic deaths from 12 districts. In the lockdown period, these 12 districts accounted for more than half $(53.4 \%)$ of the total deaths in Nepal. The largest number of people were killed in Kathmandu Valley's roads, followed by Kailali. Furthermore, Lumbini Province has the highest proportion $(20.3 \%)$ of road traffic deaths, followed by Bagmati Province (13.6\%).

The information on fatal crashes by location extracted from media reports has been visualized (Figure 2) to show the crash-prone areas of Nepal. The visualization has shown that fatal crashes were concentrated more in the middle and lower

Table 1. Distribution of the road crash incidents, vehicles involved in crashes, deaths and injuries during national level COVID-19, 82 days lockdown from police record and media reporting in Nepal.

\begin{tabular}{|l|l|l|l|l|}
\hline Places & $\begin{array}{l}\text { Vehicle } \\
\text { involved in } \\
\text { crashes }\end{array}$ & Incidents & Deaths & Injuries \\
\hline Province 1 & $179(6.9 \%)$ & $137(7.6 \%)$ & $36(14.1 \%)$ & $186(10.2 \%)$ \\
\hline Province 2 & $337(13.0 \%)$ & $261(14.5 \%)$ & $44(17.2 \%)$ & $332(18.2 \%)$ \\
\hline Bagmati & $130(5.0 \%)$ & $112(6.2 \%)$ & $26(10.2 \%)$ & $186(10.2 \%)$ \\
\hline Gandaki & $58(2.2 \%)$ & $57(3.2 \%)$ & $15(5.9 \%)$ & $70(3.8 \%)$ \\
\hline Lumbini & $308(11.8 \%)$ & $289(16.0 \%)$ & $68(26.6 \%)$ & $359(19.7 \%)$ \\
\hline Karnali & $27(1.0 \%)$ & $25(1.4 \%)$ & $15(5.9 \%)$ & $50(2.7 \%)$ \\
\hline Sudurpaschim & $66(2.5 \%)$ & $45(2.5 \%)$ & $25(9.8 \%)$ & $61(3.3 \%)$ \\
\hline $\begin{array}{l}\text { Kathmandu } \\
\text { Valley }\end{array}$ & $1497(57.5 \%)$ & $875(48.6 \%)$ & $27(10.5 \%)$ & $580(31.8 \%)$ \\
\hline $\begin{array}{l}\text { Total of } \\
\text { Nepal Police }\end{array}$ & $\mathbf{2 6 0 2 ( 1 0 0 \% )}$ & $\mathbf{1 8 0 1 ( 1 0 0 \% )}$ & $\mathbf{2 5 6 ( 1 0 0 \% )}$ & $\mathbf{1 8 2 4 ( 1 0 0 \% )}$ \\
\hline Daily average & $\mathbf{3 1 . 7}$ vehicles & $\mathbf{2 2 . 0}$ incidents & $\mathbf{3 . 1}$ deaths & $\mathbf{2 2 . 2}$ injuries \\
\hline $\begin{array}{l}\text { Media } \\
\text { reported }\end{array}$ & $\mathbf{5 4 4}$ & $\mathbf{2 7 2}$ & $\mathbf{2 0 0}$ & $\mathbf{3 2 2}$ \\
\hline Soure: Nepal & & & & \\
\hline
\end{tabular}

Source: Nepal Police Province 1, 2, Bagmati, Gandaki, Lumbini, Karnali and Sudurpaschim headquarters, Metropolitan Traffic Police Office and media reports of road crashes reported 


\section{Table 2. Districts with highest number of road traffic deaths in Nepal during the COVID-19 lockdown.}

\begin{tabular}{|l|l|l|l|}
\hline District & Province & $\begin{array}{l}\text { Number } \\
\text { of } \\
\text { deaths }\end{array}$ & Percentage \\
\hline Kathmandu Valley* & Bagmati & 27 & 10.5 \\
\hline Kailali & Sudurpaschim & 20 & 7.8 \\
\hline Banke & Lumbini & 19 & 7.4 \\
\hline Nawalparasi West & Lumbini & 13 & 5.1 \\
\hline Morang & Province 1 & 11 & 4.3 \\
\hline Siraha & Province 2 & 11 & 4.3 \\
\hline Dang & Lumbini & 11 & 4.3 \\
\hline Rautahat & Province 2 & 9 & 3.5 \\
\hline Rupandehi & Lumbini & 9 & 3.5 \\
\hline Sindhuli & Bagmati & 8 & 3.1 \\
\hline $\begin{array}{l}\text { Subtotal of deaths } \\
\text { in 12 districts }\end{array}$ & & 138 & 53.9 \\
\hline $\begin{array}{l}\text { Total deaths in } \\
\text { Nepal }\end{array}$ & & 256 & 100.0 \\
\hline Soure: & & & \\
\hline
\end{tabular}

Source: Nepal Police Province 1, 2, Bagmati, Lumbini and Sudurpaschim headquarters record for road crashes incidents. *Kathmandu Valley comprise three districts (Kathmandu, Bhaktapur and Lalitpur) region of country. Regarding provinces, the fatal crashes were higher in various locations of Bagmati Province and Lumbini Province. The visualization additionally demonstrates that a large number of fatal crashes have occurred in local units in the junction of national highways of different local government units.

Table 3 shows the type of vehicles involved in crashes and the objects, animals or people they collided with. Motorcycles were the most common vehicles involved in fatal crashes, as usual. Among the total vehicles involved in the crashes, more than one-fifth $(22.1 \%)$ were motorcycles followed by jeeps, tractors, and trucks. Along with other vehicles, 20 ambulances were found to be involved in road crashes which either hit other vehicles, people, animals or roadside objects. The majority of the vehicles involved in the crashes were reportedly out of the driver's control $(52.2 \%)$ due to speeding. Similarly, 37 pedestrians were hit by vehicles in these 82 days. Motorcyclists, pedestrians and cyclists were the most at-risk road users during road users from motorcyclists and the vehicles at essential services i.e. jeep/cars, tractors, trucks and ambulances (Table 3).

Comparing the police data for lockdown and same period last year shows a considerable reduction in the number of incidents, involved vehicles and casualties (Figure 3). Given the lower number vehicles allowed to operate the figures are still high.

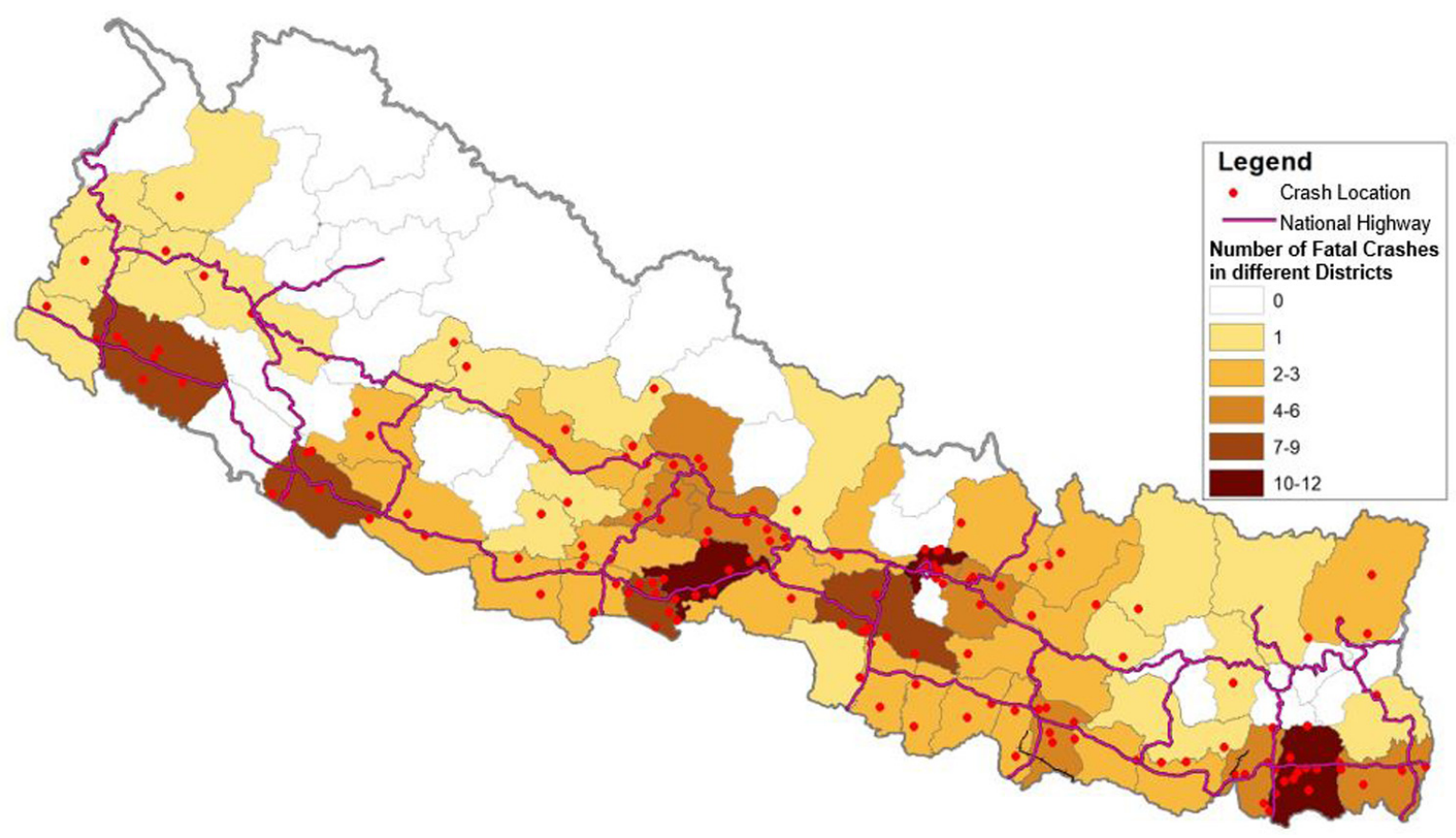

Figure 2. Visualization of road crashes deaths during COVID-19 lockdown reported in the media by Palika and national highways. Source: Locations of road crashes and fatalities from media reports. Map reproduced with the permission of the Survey Department of Nepal (2020) (Nepal Government Survey Department, 2020). 


\begin{tabular}{|c|c|c|c|c|c|}
\hline Vehicles involved & & & Counterparts & & \\
\hline Type & Number & Percent & Type & Number & Percent \\
\hline Ambulance & 20 & 7.4 & Animal & 1 & 0.4 \\
\hline Cyclist & 3 & 1.1 & Auto tempo & 1 & 0.4 \\
\hline Bus & 10 & 3.7 & Cyclist & 9 & 3.3 \\
\hline Excavator & 1 & 0.4 & Car & 3 & 1.1 \\
\hline HDV & 1 & 0.4 & Jeep & 4 & 1.5 \\
\hline Jeep & 54 & 19.9 & Motorcycle & 40 & 14.7 \\
\hline Motorcycle & 76 & 27.9 & Lamppost & 3 & 1.1 \\
\hline Power trailer & 2 & 0.7 & Pedestrian & 37 & 13.6 \\
\hline Tanker & 2 & 0.7 & Tractor & 4 & 1.5 \\
\hline Tipper & 10 & 3.7 & Tanker & 1 & 0.4 \\
\hline Tractor & 52 & 19.1 & Tree & 2 & 0.7 \\
\hline Truck & 34 & 12.5 & Truck & 6 & 2.2 \\
\hline Unidentified & 5 & 1.8 & $\begin{array}{l}\text { None } \\
(\text { Uncontrolled*) }\end{array}$ & 142 & 52.2 \\
\hline Van & 2 & 0.7 & Unidentified & 19 & 7.0 \\
\hline Total & 272 & 100.0 & Total & 272 & 100.0 \\
\hline
\end{tabular}

*'uncontrolled' is the term when it is reported 'the vehicle went beyond the control of the driver'

\section{Discussion}

Since testing for COVID-19 cases commenced in Nepal, 5,760 positive cases (as of 14 June 2020) and 19 deaths have been identified (Worldometer, 2020). Meanwhile, 256 deaths and 1,824 injuries from road crashes were recorded between 24 March to 14 June 2020.

Road traffic injuries are the leading cause of death for the people aged 5-29 years worldwide (World Health Organization, 2018). It is also the leading cause of death and disabilities among people aged between 15 and 49 years in Nepal (Pant et al., 2020). Regardless of the figures, which may vary from source to source, we aim to highlight road safety measures and their importance for the essential-service vehicles during adversity.

The total burden of road traffic injuries in Nepal is calculated to be approximately 123 million USD for 2017 (Banstola et al., 2020). The amount of indirect cost of road traffic injuries ranges from $51 \%$ in Iran to $90 \%$ in Nepal (Banstola et al., 2020; Rezaei et al., 2014). The toll of road crash deaths has demonstrated the economic impact of road crashes in Nepal. Two-wheeled motorized vehicles (motorcycles and scooters) were most frequently involved in crashes and are found to be putting the largest burden on the economy directly and indirectly (Sapkota et al., 2016). Tractors and jeeps were the second-most frequently involved vehicles in road crashes, which is shown by both the police and media records. An incident of injury tends to become a matter of interest to the media even in an adverse situation. Therefore, not all incidents of road crashes are covered by media. From our data, it is also apparent that the fatal cases are consistently reported in police records and media reports but cases of injuries are much less reported by the media.

From police records, an average of 154 incidents of road crashes took place weekly, killing 22 people and causing 156 injuries during the period of lockdown. In the normal situation, 7.6 people die in the road crashes (Nepal Police, 2019) and whereas in this lockdown even with the minimal transport mobility, on average 3.1 people died per day in Nepal. Comparing the police data for lockdown and same period in previous years, it shows considerable reduction in the number of incidents, involved vehicles and casualties (Figure 3). Given the lower traffic volume during lockdown, these figures can be considered to be high. Similarly, the ratio of deaths and injuries has spiked (in Kathmandu valley) from 1 death per 46.3 injuries during the non-lockdown period ((Police Records 2019), to 1 death per 20.6 injuries in lockdown. Nationally, the number of casualty (deaths and injuries combined) per 100 


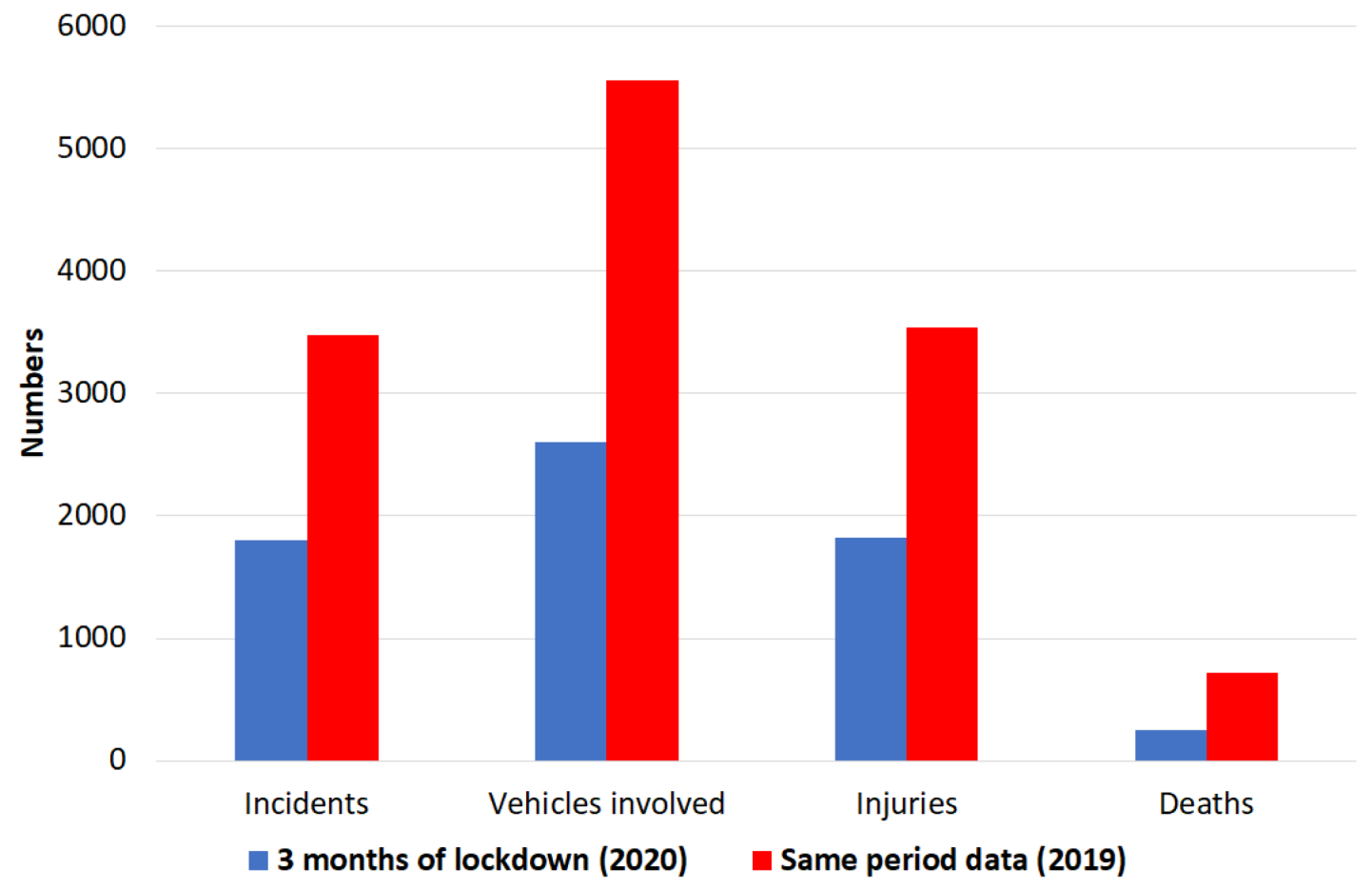

Figure 3. Comparison of incidents of road crashes, injuries and deaths during the 3 months of COVID-19 lockdown and the same period in 2019.

vehicles (involved in road crashes) was 79.9 compared to 76.5 the same period last year. Perhaps the injured individuals were involved in more severe crashes during lockdown due to people's tendency to maintain higher speeds on the road. Drivers want to drive their vehicles at high speeds for different reasons (Gabany et al., 1997), and when the roads are empty, speeding might become obvious if there are no measures in place for speed control.

\section{What do the data indicate?}

The casualty data indicate that the burden of road crashes remains high in the lockdown period, a discovery that is different from a popular belief that causality or crashes have decreased substantially. In the absence of evidence-based practice of road safety, people incorrectly assume that reduced vehicular movement automatically reduces the risks of a crash. Given the small number of vehicles in operation, the problem is rather big. This increased number of deaths and injuries during the lockdown in Nepal can be related to higher speed due to lower traffic volume and limited law enforcement. High speed means higher impact if there is a crash. It has also reported elsewhere that speed law violations and failing to stop due to high speeding were increased during lockdown (Inada et al., 2021).

These crash and casualty figures worryingly indicating the magnitude of the problem when regular transportation will eventually resume in Nepal. In rural areas, the use of tractors on unsafe roads increases the risks of crashes. Further, our findings also indicate the lack of a safety culture among the operators of the essential services (including ambulances and the vehicles used by law enforcers). The current focus of the government is to improve roads, but free roads encourage drivers to speed, which is dangerous in terms of road crashes. Therefore, a system of speed monitoring must also be integrated.

\section{Conclusion}

Roadways are the major means of transport in Nepal. Just before this lockdown, a large number of people had to make journeys to their homes (mainly going out of Kathmandu and coming to Nepal from bordering India) by roads; many of those were exposed to the risks of unsafe roads transportation which led to their deaths and injuries. Our study found that deaths on Nepali roads was not stopped during lockdown. Comparing the pattern of road crashes during the same period last year, lockdown witnessed almost half of the number of incidents $(1,801$ vs 3,480) and the number of vehicles involved in crashes $(2,602$ vs 5,560$)$. When comparing the statistics with the situation with the three months before the lockdown, it was observed that the percentages of tractors, trucks/tankers and cyclists was higher (published police records). In cities, traffic congestion was eased during the lockdown which consequently resulted higher speed, increasing the chances and 
impact of crashes. Therefore, awareness of safety and taking into account road and weather conditions when deciding to take a journey would help to keep people safe on the roads. Therefore, this lockdown has reinforced how important the management of safer mobility issue is in Nepal. Interestingly, some of the preventative measures that have been proven effective to decelerate the spread of coronavirus apply in the context of road safety as these measures can teach us something for the road safety epidemic as well (Job, 2020). The Government of Nepal has mobilised unprecedented amount of resources in terms of human resources, budget and materials to address COVID-19 which has kept the rates of infection and deaths at minimum. If similar efforts and investments are done to address the problem of road traffic injuries, it would be possible to reverse the trend of ever-increasing burden of road injuries.

\section{Data availability}

Underlying data

Figshare: Road Traffic Injuries in Nepal during COVID-19 Lockdown_ Media reporting and Police record (24 March to 14 June, 2020).csv. https://doi.org/10.6084/m9.figshare.12958373.v3 (Sedain \& Pant, 2020).
This project contains the following underlying data:

- Road Traffic Injuries in Nepal during COVID-19 Lockdown_Media reporting (24 March to 14 June, 2020).csv. (Road traffic injuries and deaths reported by local media.)

- Road Traffic Injuries in Nepal during COVID-19 Lockdown_Police records (24 March to 14 June, 2020). xlsx.csv. (Road traffic injuries and deaths taken form police records.)

Data are available under the terms of the Creative Commons Attribution 4.0 International license (CC-BY 4.0).

\section{Acknowledgments}

We would like to thank Mr. Santosh Sapkota, Metro Traffic FM, Nepal Police Human Resource and Administration Department for their valuable support. Similarly, I would like to thank Mr. Abhasha Joshi of Department of Survey for his technical support in plotting the locations of road crashes on the map of Nepal.

\section{References}

Asian Development Bank: The Economic Impact of the COVID-19 Outbreak on Developing Asia. 2020; 9(128)

Publisher Full Text

Banstola A, Kigozi J, Barton $P$, et al: : Economic burden of road traffic injuries in Nepal. Int J Environ Res Public Health. 2020; 17(12): 4571.

PubMed Abstract | Publisher Full Text | Free Full Text

Bastola A, Sah R, Rodriguez-Morales AJ, et al.: The first 2019 novel coronavirus case in Nepal. Lancet Infect Dis. 2020; 20(3): 279-80.

PubMed Abstract | Publisher Full Text | Free Full Text

Budhathoki A: COVID-19 Imperils Nepal's high economic ambitions. THE DIPLOMAT. 2020.

Reference Source

Dhakal S, Karki S: Early Epidemiological Features of COVID-19 in Nepal and

Public Health Response. Front Med (Lausanne). 2020; 7: 524.

PubMed Abstract | Publisher Full Text | Free Full Text

Embassy of Nepal, B: Address to the nation by right honourable prime minister Mr. KP Sharma Oli on control and prevention of coronavirus. 2020; Retrieved March 26, 2020.

Reference Source

Gabany GS, Plummer P, Grigg P: Why drivers speed: The speeding perception inventory. J Safety Res. 1997; 28(1): 29-35.

Publisher Full Text

Inada $\mathrm{H}$, Ashraf $\mathrm{L}$, Campbell S: COVID-19 lockdown and fatal motor vehicle collisions due to speed-related traffic violations in Japan: a time-series study. Inj Prev. 2021; 27(1): 98-100.

PubMed Abstract | Publisher Full Text

Job S: Can COVID-19 teach us something for the road safety epidemic? 2020. Reference Source

Kharel D: Nepal plans to open in five stages: Government mulls phase-wise easing of lockdown after 14 June. Nepali Times. 2020.

Reference Source

Mahato $P$, Tamang $P$, Simkhada $P$, et al.: Effects of COVID-19 during lockdown in Nepal. Europasian J Med Sci. 2020; 2(2): 1-11.

Publisher Full Text

Nepal Government Survey Department: Nepal's (political and administrative) map, Shapefile (GIS Data). 2020; Retrieved June 15, 2020.

Reference Source

Nepal Police: Police mirror 2019. 2019; Kathmandu.

Reference Source
Pant PR, Banstola A, Bhatta S, et al.: Burden of injuries in Nepal, 1990 - 2017 : findings from the Global Burden of Disease Study 2017. Inj Prev. 2020; 26(Supp 1): i57-i66.

PubMed Abstract | Publisher Full Text

Pokhrel M, Awale S: Returnees may be taking coronavirus to rural Nepal. Nepali Times. 2020.

Reference Source

Poudel A: Nepal reports its first Covid-19 death. Kathmandu Post. 2020

Reference Source

Rezaei S, Arab M, Karami MB, et al.: Extent, consequences and economic burden of road traffic crashes in Iran. J Inj Violence Res. 2014; 6(2): 57-63. PubMed Abstract | Publisher Full Text | Free Full Text

Sapkota D, Bista B, Adhikari SR: Economic costs associated with motorbike accidents in Kathmandu, Nepal. Front Public Health. 2016; 4: 273.

PubMed Abstract | Publisher Full Text | Free Full Text

Sedain B, Pant PR: Road Traffic Injuries in Nepal during CoVID-19 Lockdown Media reporting and Police record (24 March to 14 June, 2020). csv. figshare. Dataset. 2020.

http://wwww.doi.org/10.6084/m9.figshare.12958373.v3

Wang $Y$, Yuan $Y$, Wang $Q$, et al.: Changes in air quality related to the control of coronavirus in China: Implications for traffic and industrial emissions. Sci Total Environ. 2020; 731: 139133.

PubMed Abstract | Publisher Full Text | Free Full Text

World Health Organization: Infection prevention and control of epidemicand pandemic-prone acute respiratory infections in health care. 2014 Geneva.

Reference Source

World Health Organization: Global status report on road safety 2018. 2018; Geneva.

Reference Source

World Health Organization: Infection prevention and control of epidemicand pandemic-prone acute respiratory infections in health care. 2020 Geneva.

Reference Source

Worldometer: Coronavirus worldwide graphs. 2020; Retrieved June 15, 2020 Reference Source

Zhu W, Yang J, Xu L, et al: A plunge in the number of traumatic traffic injuries in an emergency center in Anhui province, China. Am J Emerg Med. 2020; 38(10): 2230-2231.

PubMed Abstract | Publisher Full Text | Free Full Text 


\section{Open Peer Review}

\section{Current Peer Review Status:}

\section{Version 3}

Reviewer Report 02 February 2021

https://doi.org/10.5256/f1000research.54194.r78581

(C) 2021 Siebert F. This is an open access peer review report distributed under the terms of the Creative Commons Attribution License, which permits unrestricted use, distribution, and reproduction in any medium, provided the original work is properly cited.

\section{Felix Wilhelm Siebert}

Friedrich Schiller University Jena, Jena, Germany

No further comments.

Competing Interests: No competing interests were disclosed.

Reviewer Expertise: Traffic psychology

I confirm that I have read this submission and believe that I have an appropriate level of expertise to confirm that it is of an acceptable scientific standard.

\section{Version 2}

Reviewer Report 20 January 2021

\section{https://doi.org/10.5256/f1000research.31067.r76068}

(C) 2021 Siebert F. This is an open access peer review report distributed under the terms of the Creative Commons Attribution License, which permits unrestricted use, distribution, and reproduction in any medium, provided the original work is properly cited.

\section{Felix Wilhelm Siebert}

Friedrich Schiller University Jena, Jena, Germany

The authors have addressed my major and minor comments from the first revision. Only two minor comments remain:

For Figure 3, the authors should add a y-axis label and should consider relabeling the bars as "3 months of lockdown" and "prior year data (2019)". Potentially the Figure description 
should be revised to "Comparison of incidents of road crashes, injuries and deaths during the 3 months COVID-19 lockdown and the same time frame in the year before".

Figure 3 indicates that there is indeed a decrease in the number of incidents, injuries and deaths during the lockdown in comparison to the same time frame in 2019. Hence, I would suggest to slightly reword the abstract, to more clearly state that a reduction was found. This could be done by changing the order of the first sentence of the conclusion, along the lines of: "While a reduction in the number of road crashes and related injuries and fatalities was found, this reduction was not as substantial as could have been expected due to the heavy movement restrictions imposed during the lock down". (just a suggestion).

Competing Interests: No competing interests were disclosed.

Reviewer Expertise: Traffic psychology

I confirm that I have read this submission and believe that I have an appropriate level of expertise to confirm that it is of an acceptable scientific standard.

Reviewer Report 18 January 2021

https://doi.org/10.5256/f1000research.31067.r76067

(C) 2021 Mani K. This is an open access peer review report distributed under the terms of the Creative Commons Attribution License, which permits unrestricted use, distribution, and reproduction in any medium, provided the original work is properly cited.

\section{Kulanthayan KC Mani}

Safe Kids Malaysia, Department of Community Health, Faculty of Medicine and Health Sciences, University Putra Malaysia (UPM), Serdang, Malaysia

The authors have clarified the concerns raised and understanding the limitation during the pandemic, the best available data on RTC is to be obtained from media source compared to police data. With this statement stated in the article, the article is fine. Thanks for considering the points raised. Best wishes.

Competing Interests: No competing interests were disclosed.

Reviewer Expertise: Injury prevention and safety promotion

I confirm that I have read this submission and believe that I have an appropriate level of expertise to confirm that it is of an acceptable scientific standard.

Version 1 
Reviewer Report 10 November 2020

https://doi.org/10.5256/f1000research.29008.r72705

(C) 2020 Siebert F. This is an open access peer review report distributed under the terms of the Creative Commons Attribution License, which permits unrestricted use, distribution, and reproduction in any medium, provided the original work is properly cited.

\section{Felix Wilhelm Siebert}

Friedrich Schiller University Jena, Jena, Germany

The study presents secondary source road traffic injury and fatality data from Nepal for the time frame of the recent COVID-19 related lockdown between March and June 2020.

I understood the study as follows: The authors used Nepal Traffic Police data to extract numbers of fatalities and injuries (distinguished between heavy and non-heavy injuries) for the timeframe between March and June 2020. In addition, news articles were analyzed and reports on injuries and fatalities were collected for the same timeframe. Both datasets are then compared, and the authors find that not all fatal crashes are reported on, while even less of a percentage of crashes that "only" result in injuries are reported in the news. The authors list potential factors related to accident causes and hypothesize that speeding might play a larger role in crashes during the lockdown, as the road system is less crowded.

The article is very easy to read and all data is presented in a concise and clear way. I have two major comments before going through the manuscript in detail.

\section{Comparison to pre-lockdown data}

Before reading the manuscript, I expected that the authors would compare injury and fatality data during the lockdown to data collected outside of the lockdown period, e.g. to the same days in 2019. This would give an indication if the lockdown has led to a general increase in fatalities and injuries (this would be expected). The authors report non-lockdown data in the discussion section of the article (p.5 "In the normal situation 7.6 people die in the road crashes [...]") but I would have expected this comparison sooner, i.e. in the results section.

In addition, the comparison of the ratio of injuries to deaths should be presented sooner than in the discussion. The ratio named for the non-lockdown period ("Similarly, the ratio of deaths and injuries - a number that has surged from 1 death per 22.8 injuries during the non-lockdown period (Karkee \& Lee, 2016), to 1 death per 7.1 injuries in lockdown.") is old, as the data used in the Karkee \& Lee (2016) paper is from 2013.

Furthermore, it's unclear if the correct data from the Karkee and Lee paper is used to arrive at the death/injury ratio. It appears that the ratio of 1 death per 22.8 injuries is calculated from Table 1 in the cited article, but erroneously only injury and fatality data for Kathmandu is used. Calculating the death/injury ratio from the correct column (listing the "total" number for the last year of available data) results in a death/injury ratio of 6.6. Hence, I advise that the authors either make it more clear which data is used for the calculation of the ratio, or (preferably) calculate the more current ratio for the whole year 2019 and for the exact same time frame as the 2020 lockdown, using 2019 data.

\section{Timely distribution of fatalities and injuries within the lockdown time frame}

The authors do a great job in describing outside factors that have influenced traffic density during 
the lockdown, i.e. especially before the lockdown there seems to have been higher traffic as people left for their home villages / home countries. While reading, I was wondering if this potential uneven distribution of traffic within the observed time frame would be visible in the distribution of injuries and fatalities, e.g. more injuries and fatalities in the beginning of the lockdown than in the middle/end. This could be visualized easily by plotting the percentage of registered injuries and fatalities over the lockdown time frame. This could also be presented alongside the same data for the same time frame in 2019.

\section{Other comments:}

The title omits the analysis of fatalities during the lockdown, the authors should consider revising the title "Road traffic injuries and fatalities during [...]"

I understand the urge to contrast numbers of road related fatalities with COVID-19 numbers, but they are very different things, mainly as they have completely different potential for exponential increases. Hence, I would suggest the authors revise the first sentence of the abstract to make road safety and COVID less contrastive.

The results section of the abstract should present comparative data to the same time frame from a non-lockdown period.

The conclusion part of the abstract is not supported by the results part of the abstract. The sentence "Even when the movement restrictions were imposed in Nepal, the number of road crashes was not substantially reduced." needs to be supported by additional analyses in the manuscript, which will need to be mentioned in the results part of the abstract.

"Within the window of the partial lockdown" please list clear time frames (i.e. dates).

"Meanwhile, the second case of COVID-19 was detected on 23 March." when was the first case detected? The authors should consider adding a timeline of COVID-related events in Nepal (e.g. First case, partial lockdown, lockdown, opening up, analyzed time frame).

Do the authors have any information on the number of special "pass-permits" handed out during the lockdown? This could be related to the number of registered vehicles in Nepal, to get an idea of how much the traffic was reduced through the lockdown (hypothetically).

In the discussion section, I would suggest the authors do not directly contrast COVID-19 and road related fatalities (same argument as before). It might be enough to change "On the contrary $[\ldots]^{\prime \prime}$ to a different wording.

The second and third section in the discussion ("Road traffic injuries are the leading cause of death for $\left.[. . .]^{\prime \prime}\right)$ are quite general and do not directly relate to the results found in the study. I think they are better suited to frame road safety challenges (global and in Nepal), and would be better placed in the introduction.

For the sentence "Similarly, the ratio of deaths and injuries - a number that has surged from 1 death per 22.8 injuries during the non-lockdown period (Karkee \& Lee, 2016), to 1 death per 7.1 injuries in lockdown." Please revise for grammar. (and please also see my first major comment above). 
The section "What do the data indicate? The casualty data indicate that the burden of road crashes remains high in the lockdown period [...]" is not well supported by the results section of the paper (see my first major comment).

In the conclusion, the authors write "Roadways are the major means of transport in Nepal. In this lockdown, large number of people had to make journeys to their homes by roads [...]". But the authors earlier state, that the main movement of people happened during partial lockdown, i.e. before the time frame analyzed in this study. One could assume that during the early days of lockdown, some people used their vehicles illegally (without the special pass-permit), but this would need to be stated more clearly. The authors should clear this up (see also my second major comment).

Is the work clearly and accurately presented and does it cite the current literature? Yes

Is the study design appropriate and is the work technically sound? Partly

Are sufficient details of methods and analysis provided to allow replication by others? Yes

If applicable, is the statistical analysis and its interpretation appropriate? Partly

Are all the source data underlying the results available to ensure full reproducibility? Yes

Are the conclusions drawn adequately supported by the results? Partly

Competing Interests: No competing interests were disclosed.

Reviewer Expertise: Traffic psychology

I confirm that I have read this submission and believe that I have an appropriate level of expertise to confirm that it is of an acceptable scientific standard, however I have significant reservations, as outlined above.

Author Response 26 Nov 2020

Bhagabati Sedain, Padma Kanya Multiple Campus, Tribhuvan University, Kathmandu, Nepal

Comparison to pre-lockdown data

Before reading the manuscript, I expected that the authors would compare injury and fatality data during the lockdown to data collected outside of the lockdown period, e.g. to 
the same days in 2019. This would give an indication if the lockdown has led to a general increase in fatalities and injuries (this would be expected). The authors report non-lockdown data in the discussion section of the article (p.5 "In the normal situation 7.6 people die in the road crashes [...]") but I would have expected this comparison sooner, i.e. in the results section.

In addition, the comparison of the ratio of injuries to deaths should be presented sooner than in the discussion. The ratio named for the non-lockdown period ("Similarly, the ratio of deaths and injuries - a number that has surged from 1 death per 22.8 injuries during the non-lockdown period (Karkee \& Lee, 2016), to 1 death per 7.1 injuries in lockdown.") is old, as the data used in the Karkee \& Lee (2016) paper is from 2013.

Furthermore, it's unclear if the correct data from the Karkee and Lee paper is used to arrive at the death/injury ratio. It appears that the ratio of 1 death per 22.8 injuries is calculated from Table 1 in the cited article, but erroneously only injury and fatality data for Kathmandu is used. Calculating the death/injury ratio from the correct column (listing the "total" number for the last year of available data) results in a death/injury ratio of 6.6. Hence, I advise that the authors either make it more clear which data is used for the calculation of the ratio, or (preferably) calculate the more current ratio for the whole year 2019 and for the exact same time frame as the 2020 lockdown, using 2019 data.

Authors' response: Thanks for cogent observations. We have addressed them as below: compare injury and fatality data during the lockdown to data collected outside of the lockdown period, e.g. to the same days in 2019.

used them accordingly. We have now replaced the terms relating to 'comparison' throughout the text.

The ratio of deaths and injuries for lockdown and non-lockdown period

Authors' response: Thank you very much for spotting the error. We appreciate your advice for using more recent figures. We compared the figures with those during the same period last year (i.e. 3 months of lockdown compared with the same 3 months last year). we have revised this statement as: ("Similarly, the ratio of deaths and injuries has surged (in Kathmandu) from 1 death per 46.3 injuries during the non-lockdown period (Police Records), to 1 death per 20.6 injuries during the lockdown." A new sentence is added "Nationally, the number of casualty (death plus injuries) per 100 involved vehicles were slightly higher (79.9) during lockdown compared to the same period last year (76.5)."

Timely distribution of fatalities and injuries within the lockdown time frame The authors do a great job in describing outside factors that have influenced traffic density during the lockdown, i.e. especially before the lockdown there seems to have been higher traffic as people left for their home villages / home countries. While reading, I was wondering if this potential uneven distribution of traffic within the observed time frame would be visible in the distribution of injuries and fatalities, e.g. more injuries and fatalities in the beginning of the lockdown than in the middle/end. This could be visualized easily by plotting the percentage of registered injuries and fatalities over the lockdown time frame. This could also be presented alongside the same data for the same time frame in 2019.

\section{Other comments:}

The title omits the analysis of fatalities during the lockdown, the authors should consider revising the title "Road traffic injuries and fatalities during [...]" 
Authors' Response: Thank you very much for your suggestion. We think the title is ok.

I understand the urge to contrast numbers of road related fatalities with COVID-19 numbers, but they are very different things, mainly as they have completely different potential for exponential increases. Hence, I would suggest the authors revise the first sentence of the abstract to make road safety and COVID less contrastive.

Authors' response: Thanks for your suggestion but we think this statement is reasonable and we would like to keep it as is.

The results section of the abstract should present comparative data to the same time frame from a non-lockdown period.

Authors' response: We agree that adding a figure with the information during non-lock down would enhance the text. Figure 3 has been added to the discussion section and cited in the fourth paragraph (p5). "Comparing the police data for lockdown and the same period last year shows a considerable reduction in the number of incidents, involved vehicles and casualties (Figure 3). Given the lower number of vehicles allowed to operate the figures are high."

The conclusion part of the abstract is not supported by the results part of the abstract. The sentence "Even when the movement restrictions were imposed in Nepal, the number of road crashes was not substantially reduced." needs to be supported by additional analyses in the manuscript, which will need to be mentioned in the results part of the abstract.

Authors Response: We have revised the conclusion, it was also suggested by reviewer 1 (please see the conclusion section in the text).

"Within the window of the partial lockdown" please list clear time frames (i.e. dates). Authors Response: 21 march to 23 march

"Meanwhile, the second case of COVID-19 was detected on 23 March." when was the first case detected? The authors should consider adding a timeline of COVID-related events in Nepal (e.g. First case, partial lockdown, lockdown, opening up, analyzed time frame).

Authors' response: The first case was confirmed on 13 th January and we have added a figure (Figure 1) in the introduction

Do the authors have any information on the number of special "pass-permits" handed out during the lockdown? This could be related to the number of registered vehicles in Nepal, to get an idea of how much the traffic was reduced through the lockdown (hypothetically). Authors' response: unfortunately, no information available in this regard

In the discussion section, I would suggest the authors do not directly contrast COVID-19 and road related fatalities (same argument as before). It might be enough to change "On the contrary [...]" to a different wording.

Authors' response: we have changed "on the contrary", as "meanwhile"

The second and third section in the discussion ("Road traffic injuries are the leading cause of death for [...]") are quite general and do not directly relate to the results found in the study. I think they are better suited to frame road safety challenges (global and in Nepal), and would be better placed in the introduction. 
Authors' response: Thanks for the suggestion, but we think these two statements are setting the context for discussion.

For the sentence "Similarly, the ratio of deaths and injuries - a number that has surged from 1 death per 22.8 injuries during the non-lockdown period (Karkee \& Lee, 2016), to 1 death per 7.1 injuries in lockdown." Please revise for grammar. (and please also see my first major comment above).

Authors' response: we have responded to it above

The section "What do the data indicate? The casualty data indicate that the burden of road crashes remains high in the lockdown period [...]" is not well supported by the results section of the paper (see my first major comment).

Authors' response: Thanks for the suggestion, we have now revised the sentence: "The casualty data indicate that the burden of road crashes remains high in the lockdown period despite of very much restricted vehicular movement"

In the conclusion, the authors write "Roadways are the major means of transport in Nepal. In this lockdown, large number of people had to make journeys to their homes by roads [...]". But the authors earlier state, that the main movement of people happened during the partial lockdown, i.e. before the time frame analyzed in this study. One could assume that during the early days of lockdown, some people used their vehicles illegally (without the special pass-permit), but this would need to be stated more clearly. The authors should clear this up (see also my second major comment).

Authors' response: Thanks for the suggestion, we have now revised the sentence as "Roadways are the major means of transport in Nepal. Just before this lockdown, a large number of people had to make journeys to their homes (mainly going out of Kathmandu and coming to Nepal from bordering India) by roads immediately after the announcement of the lockdown".

Competing Interests: No competing interests

Reviewer Report 29 October 2020

https://doi.org/10.5256/f1000research.29008.r72710

(C) 2020 Mani K. This is an open access peer review report distributed under the terms of the Creative Commons Attribution License, which permits unrestricted use, distribution, and reproduction in any medium, provided the original work is properly cited.

\section{Kulanthayan KC Mani}

Safe Kids Malaysia, Department of Community Health, Faculty of Medicine and Health Sciences, University Putra Malaysia (UPM), Serdang, Malaysia

1. Abstract - For the methods sections, usage of secondary data from Police is fine and acceptable, but not from the media reports. Media reports does not cover all crashes and there could also be duplication of a single case is being reported by various media. Also it's 
the reporter and editor view. No one to verify it. Not recommended for scientific article unless no other options.

2. Methods - is it possible to do away with the media reports and completely rely on the police data (secondary data)? What kind of information we can get from police? If that is sufficient, then go ahead with police data only.

3. Results. Table 1. The paragraph tend to indicate as though the article is trying to compare two reporting system police vs media which is not the case as I believe you are using media to complement and close the gap for info not available from police source. Intention good, however the mechanism to address it is questionable since its unverified and its individually done by reporters.

4. Table 2. Purpose of it? My understanding is based on the title, there could be two options of story flow: 1. Before and during covid: RTC and RTI status. Option 2: study RTC and RTI during covid (there must be a purpose and benefit of doing one). Till this stage not clear yet.

5. Figure 1. Purpose of it? Does it tell us during covid the mapping of RTC and RTI are different compared to non-covid time. Therefore this are the areas to be targeted and focused by the government towards addressing the problem. Is this what you intend to?

6. Table 3. Purpose of it? Any different on its distribution / patterns from normal day which warrants attention? How best can we use this info towards our next move in terms of strategies or policy change with an aim to reduce RTC and RTI during covid.

7. Did we learn anything during covid which we can put to use after this which can give us better results in terms of managing RTC and RTI - example physical distancing, reducing exposure, lower ridership in vehicle etc. Effect of covid could be shift in travel mode to private vehicles or single rider vehicles to avoid crowd. Vehicle speed increases as more space with lower vehicle. There could be reductions in RTC, but RTI Fatal may not drop much.

8. Discussion para 3. USD123 million is for which year estimates? Interestingly $90 \%$ is based on indirect cost. Compared to other country studies, what are their range of $\%$ for indirect cost? Is it as high as this $90 \%$ ?

9. What do the data indicate? RTC and RTI during covid should be compared during normal day within Nepal also. That is the right comparison and not against other countries like Fiji, Estonia, Cyprus etc.

10. Interesting to note on crashes involving ambulances during covid in comparison to normal day. Are they at more risk? If yes, what need to be done next? Increase in tractor usage does it adds to more crash? Any police data to show increase? Generally tractor as low speed vehicle pose less harm. Its only risk is when it shares the roadway with mixed traffic and the other traffic speed is very high and huge difference with the tractor speed.

11. Conclusion - the content in conclusion is very much different compared to the text inside the article. Many aspects not touched in the article is being concluded here example of 
using PPE, safety helmet, seat belts and CRS. Prefer conclusion strictly based on this study findings.

Is the work clearly and accurately presented and does it cite the current literature? Yes

Is the study design appropriate and is the work technically sound? Partly

Are sufficient details of methods and analysis provided to allow replication by others? Partly

If applicable, is the statistical analysis and its interpretation appropriate? No

Are all the source data underlying the results available to ensure full reproducibility? Partly

Are the conclusions drawn adequately supported by the results? No

Competing Interests: No competing interests were disclosed.

Reviewer Expertise: Injury prevention and safety promotion

I confirm that I have read this submission and believe that I have an appropriate level of expertise to confirm that it is of an acceptable scientific standard, however I have significant reservations, as outlined above.

Author Response 26 Nov 2020

Bhagabati Sedain, Padma Kanya Multiple Campus, Tribhuvan University, Kathmandu, Nepal

1.Abstract - For the methods sections, usage of secondary data from Police is fine and acceptable, but not from the media reports. Media reports does not cover all crashes and there could also be duplication of a single case is being reported by various media. Also, it's the reporter and editor view. No one to verify it. Not recommended for scientific article unless no other options.

Author response: Thank you for the concerns. We were aware that media reports do not cover all crashes, particularly those with non-fatal outcomes. During the period of lockdown and minimum vehicular movement, Nepali media covered $78 \%$ of all fatal incidents compared to police records (Table 1). We extracted detailed information for the individual crashes from media reports. A typical Nepali media report of a road crash, it reports - location of the crash; vehicle type and registration number; gender and age of victims (with names); outcomes of the crash. The details provided in the report obviously helped to see any duplication of the records. Such information was 
not possible to get from police records; the police source only provides compiled reports of incidents.

We have revised the last sentence under "Methods" in the abstract.

2.Methods - is it possible to do away with the media reports and completely rely on the police data (secondary data)? What kind of information we can get from police? If that is sufficient, then go ahead with police data only.

Author response: Using police data only will limit the information and we will not be able to make Figure 1 and Table 3, which is derived from media report data. We have amended the last sentence of the section Methods as - "In this study, we have presented the results from the two data sources to provide the most possible details complemented by one-another."

3.Results. Table 1. The paragraph tends to indicate as though the article is trying to compare two reporting system police vs media which is not the case as I believe you are using media to complement and close the gap for info not available from police source. Intention good, however the mechanism to address it is questionable since its unverified and its individually done by reporters.

Author response: Thank you for the comment. We have revised our statement and removed the term 'compare' throughout the text because these two data sources complement one another. However, we have discussed the differences in the variables obtained from the two sources.

4.Table 2. Purpose of it? My understanding is based on the title, there could be two options of story flow: 1. Before and during covid: RTC and RTI status. Option 2: study RTC and RTI during covid (there must be a purpose and benefit of doing one). Till this stage not clear yet. Author response: Thank you for the suggestion. We described the data as "option 2" in your comment; the purpose was to show the districts that were the most affected. We have now revised the title of table 2 "Table 2. Districts with the highest number of road traffic deaths in Nepal during national level COVID-19 lockdown." We have replaced "Province 5 " with its official name, Lumbini which is given after we submitted the manuscript.

5.Figure 1. Purpose of it? Does it tell us during covid the mapping of RTC and RTI are different compared to non-covid time? Therefore, this are the areas to be targeted and focused by the government towards addressing the problem. Is this what you intend to? Author response: The map (figure 1) shows the locations of road crashes that occurred during the lockdown. Therefore, it was not possible to compare the numbers before and during the lockdown. This information is derived from news reports, as the police data used in this study does not tell the location of crashes. We believe this map is a novel idea to show the locations of fatal road crashes and this would help relevant authorities to focus interventions.

6.Table 3. Purpose of it? Any different on its distribution / patterns from normal day which warrants attention? How best can we use this info towards our next move in terms of strategies or policy change with an aim to reduce RTC and RTI during covid.

Author response: 1 ) the purpose of table 3 was to convey to the readers what type of vehicles were involved in road crashes; and the counterpart in case it was a collision. This information was extracted from media reports because this information was not available from police data used in this paper. We have made slight changes to the title of Table 3 as "Distribution of road crashes during national level covid-19 lockdown 
according to the types of vehicles involved and their counterparts." The key message here is - Motorcyclists, pedestrians and cyclists are the most at-risk road users from motorcyclists and the vehicles at essential services i.e. jeep/cars, tractors, trucks, and ambulances. Therefore, the enforcement of road safety laws must be of priority always.

7.Did we learn anything during covid which we can put to use after this which can give us better results in terms of managing RTC and RTI - example physical distancing, reducing exposure, lower ridership in vehicle etc. Effect of covid could be shift in travel mode to private vehicles or single rider vehicles to avoid crowd. Vehicle speed increases as more space with lower vehicle. There could be reductions in RTC, but RTI Fatal may not drop much.

Author response: It is beyond the scope of this study, we only studied the period of lockdown where all non-essential travel was prohibited.

8.Discussion para 3. USD123 million is for which year estimates? Interestingly $90 \%$ is based on indirect cost. Compared to other country studies, what are their range of $\%$ for indirect cost? Is it as high as this $90 \%$ ?

Author response: Thanks for your comment. As mentioned in the reference cited in this paper, higher indirect costs are also seen in other countries. We have revised the sentences with additional reference: "The total burden of road traffic injuries in Nepal is calculated to be approximately 123 million USD for 2017, and 90\% of this amount comprised of indirect costs (Banstola et al., 2020). The amount of indirect cost of road traffic injuries ranges from $51 \%$ in Iran to $90 \%$ in Nepal (Rezaei, Arab, Karami, \& Akbari Sari, 2014; Banstola et al., 2020). This road crashes deaths toll demonstrated the economic impact of road crashes is bigger in Nepal."

9.What do the data indicate? RTC and RTI during covid should be compared during normal day within Nepal also. That is the right comparison and not against other countries like Fiji, Estonia, Cyprus etc.

Author response: Thank you very much for the comment. We have revised this section by adding the following text -

"This increased number of deaths and injuries during the lockdown in Nepal can be related to higher speed due to lower traffic volume and limited law enforcement. High speed means higher impact if there is a crash. It has also reported elsewhere that speed law violations and failing to stop due to $\quad$ high speeding were increased during lockdown (Inada et al, 2020)."

10.Interesting to note on crashes involving ambulances during covid in comparison to normal day. Are they at more risk? If yes, what need to be done next? Increase in tractor usage does it adds to more crash? Any police data to show increase? Generally, tractor as low speed vehicles pose less harm. Its only risk is when it shares the roadway with mixed traffic and the other traffic speed is very high and huge difference with the tractor speed. Author response: Thank you very much for the comment. a) Road crashes involving Ambulances are something that is not explored yet. Classification and standardization of ambulances in Nepal are yet evolving. Ambulances are a very important part of the post-crash response (pillar 5) aspect of road safety. Ambulances in Police records are not separately recorded and collectively included with other four-wheeled light vehicles (under jeep cars). Therefore, we cannot say whether there was an increase in ambulance movement or crashes during the COVID-19 lockdown. b) the use of tractors was increased for transportation of relief-aid materials (food, 
quarantine construction materials, etc) during the COVID-19 lockdown. It is true that the mix of vehicles with different speeds caused crashes; also, tractors from rural areas and hilly tracks were also reported.

11.Conclusion - the content in conclusion is very much different compared to the text inside the article. Many aspects not touched in the article is being concluded here example of using PPE, safety helmet, seat belts and CRS. Prefer conclusion strictly based on this study findings.

Author response: Thank you very much for the comment and suggestions. We have now revised the conclusion section.

Roadways are the major means of transport in Nepal. Just before this lockdown, a large number of people had to make journeys to their homes_mainly going out of Kathmandu and coming to Nepal from bordering India) by roads; many of those were exposed to the risks of unsafe roads transportation which led to their deaths and injuries. Our study found that deaths on Nepali roads was not stopped during lock down. Comparing the pattern of road crashes during the same period last year, lock down witnessed almost half of the number of incidents $(1,801$ vs 3,480$)$ and the number of vehicles involved in crashes $(2,602$ vs 5,560$)$. When comparing the statistics with the situation with the three months before the lock down, it was observed that the percentages of tractors, trucks/tankers and cyclists was higher (published police records). Therefore, this lockdown has reinforced how important the management of safer mobility issue is in Nepal. Interestingly, some of the preventative measures that have been proven effective to decelerate the spread of coronavirus apply in the context of road safety as these measures can teach us something for the road safety epidemic as well (Job, 2020). Therefore, for better road safety, unnecessary travels must be avoided and a safe distance should be maintained between the vehicles on the move. Likewise, the use of helmets, seatbelts, and child restraints are similar to the use of PPE, whereas the regular testing of vehicles, like COVID testing, is a must. In cities, traffic congestion was eased during the lockdown which consequently resulted higher speed, increasing the chances and impact of crashes. Therefore, awareness of safety and taking into account road and weather conditions when deciding to take a journey would help to keep people safe on the roads. Therefore, this lockdown has reinforced how important the management of safer mobility issue is in Nepal. Interestingly, some of the preventative measures that have been proven effective to decelerate the spread of coronavirus apply in the context of road safety as these measures can teach us something for the road safety epidemic as well (lob, 2020). The Government of Nepal has mobilised unprecedented amount of resources in terms of human resources, budget and materials to address COVID-19 which has kept the rates of infection and deaths at minimum. If similar efforts and investments are done to address the problem of road traffic injuries, it would be possible to reverse the trend of everincreasing burden of road injuries.

Competing Interests: No competing interests 


\section{Comments on this article}

\section{Version 2}

Reviewer Response 15 Jan 2021

Kulanthayan KC Mani, University Putra Malaysia (UPM), Serdang, Malaysia

The authors have clarified the concerns raised and understanding the limitation during the pandemic, the best available data on RTC is to be obtained from media source compared to police data. With this statement stated in the article, the article is fine. Thanks for considering the points raised. Best wishes

Competing Interests: No

The benefits of publishing with F1000Research:

- Your article is published within days, with no editorial bias

- You can publish traditional articles, null/negative results, case reports, data notes and more

- The peer review process is transparent and collaborative

- Your article is indexed in PubMed after passing peer review

- Dedicated customer support at every stage

For pre-submission enquiries, contact research@f1000.com 